\title{
Vaccine passport: A futuristic approach to combat the global spread of COVID-19 pandemic
}

\author{
Nitin Tyagi ${ }^{*}$ (D), Sukanya Gangopadhyay ${ }^{2}$ (D) Charanjeet Kaur $^{3}$ (D) \\ ${ }^{1}$ Senior Resident, Department of Biochemistry, Vardhman Mahavir Medical College \& Safdarjung Hospital, New Delhi, India \\ ${ }^{2}$ Associate Professor, Department of Biochemistry, Vardhman Mahavir Medical College \& Safdarjung Hospital,New Delhi, India \\ ${ }^{3}$ Professor, Department of Biochemistry, Vardhman Mahavir Medical College \& Safdarjung Hospital, New Delhi, India
}

\begin{tabular}{ll}
\hline Article Info & A B S T R A C T \\
\hline $\begin{array}{l}\text { Article type: } \\
\text { Letter to Editor }\end{array}$ & $\begin{array}{l}\text { As hope of normalcy has seemingly returned to the world following the } \\
\text { approval and rollout of various vaccines in many countries, the idea of } \\
\text { creating a vaccine passport which would prime the access of air travel and }\end{array}$ \\
\hline Article History: & $\begin{array}{l}\text { several public and private places to COVID-19 vaccination is thriving. A } \\
\text { Received: } 2021-02-07\end{array}$ \\
vaccepted: $2021-04-18$ & vaccine passport is a user-friendly app containing a users verifiable \\
Published: $2021-04-18$ & to officially prove that they have indeed been vaccinated on a particular date \\
& and time.
\end{tabular}

\section{* Corresponding author:}

Nitin Tyagi

Senior Resident, Department of

Biochemistry, Vardhman Mahavir

Medical College \& Safdarjung

Hospital, New Delhi, India

Email: drnitintyagi1903@gmail.com

\author{
Keywords: \\ Vaccine Passport \\ COVID-19 \\ Vaccination
}

\section{Cite this paper as:}

Tyagi N, Gangopadhyay S, Kaur C. Vaccine Passport: A Futuristic Approach to Combat the Global Spread of COVID-19

Pandemic. Front Health Inform. 2021; 10: 72. DOI: 10.30699/fhi.v10i1.277

\section{DEAR EDITOR}

With the introduction of COVID-19 vaccine, a new ray of hope of normalcy has seemingly returned to the world. It has led to the idea of creation of an entity called vaccine passport -which would prime the access of air travel and several public and private places to COVID-19 vaccination.

\section{What is a vaccine passport?}

A vaccine passport is an entity of user-friendly application containing a user's verifiable vaccination particulars or credentials on their mobile or other electronic devices in order to prove the recognition of being vaccinated on a particular date and time officially. The digital vaccination document often called updated version of immunity passport with the proof of vaccination, may then be utilized to gain access to work places, cinemas, public transport, restaurants, and even foreign countries.

\section{How would a vaccine passport work?}

In theory, the vaccine passport works by allowing users, airlines, laboratories, defense forces and other relevant public and private agencies access to a universal database. Depending on the need, they could then take out or place the required information from or into the database. For instance, border authorities or airlines could verify the validity of a travelers' inoculation status and the type of the administered vaccine. This would allow the authorities to obtain information on the type and efficacy of administered vaccine and act suitably.

Borders authorities or airlines won't need to do manual verification of the names of the health facility or type of administered vaccine either by phone or email. From the travelers' point of view, the vaccine passport would allow them to instantly learn of a particular nation's vaccination requirements. It may contribute to the long-term management of the COVID-19 pandemic. 


\section{Scope of vaccine passport}

Even-though there have not been any official measures for vaccine passports being executed in any country as of yet, it stands to reason that once the COVID vaccination has gained worldwide coverage, various public and private organizations may very well utilize vaccine passports as a mandatory norm for entry. In the recent time, software companies have developed digital health passport, for instance, IBM developed IBM Digital Health Pass [1] as a means to provide public and private agencies with a potent way to bring people back to the state of normalcy at workplace, school, colleges, and stadium or airline flights.

Bottom line, a point of entry-it is an international border, a concert venue, a corporate office - would want to learn of a person's inoculation status along with the accompanying variables before allowing entry. If the person has indeed been inoculated, what type of vaccine was administered, was it the Pfizer vaccine, Covishield, etc.? These variables are important as they correspond to varying efficacy rates.

\section{Current status of vaccine passport}

There are currently various groups working on development of vaccine passport apps, with significant examples being the Common Pass [ㄹ] and the International Air Transport Association (IATA) Travel Pass [ $\underline{3}]$.

The Swiss-based non-profit company The Common Projects, in collaboration with the World Economic Forum has developed The Common Pass app. The app is currently under trial phase and should be ready for global use very shortly. In the similar way, the International Air Transport Association has developed The IATA Travel Pass which is slated for a Q1 launch in the near future.

All in all, vaccination passport is a big hope of being back to normalcy via beginning of coming down curtain on the COVID-19 pandemic.

\section{AUTHOR'S CONTRIBUTION}

The authors agree on this final form of the manuscript, and attested that all authors contributed in the final draft of the manuscript.

\section{CONFLICTS OF INTEREST}

The authors declare no conflicts of interest regarding the publication of this study.

\section{FINANCIAL DISCLOSURE}

No financial interests related to the material of this manuscript have been declared.

\section{REFERENCES}

1. Eric PisCini. IBM digital health pass puts privacy first [Internet]. 2020 [cited: 25 Aug 2020]. Available from: https://www.ibm.com/blogs/watsonhealth/author/eric-pisciniibm-com

2. Hackett M. Digital health passport CommonPass begins testing to help travel and trade resume
[Internet]. 2020 [cited: 7 Oct 2020]. Available from: https://www.mobihealthnews.com/author/malloryhackett

3. IATA. Unveils key design elements of IATA travel pass [Internet]. 2020 [cited: 16 Dec 2020]. Available from: https://www.iata.org/en/pressroom/pr/2020-1216-01/ 